\title{
A Comparison between Genetic Algorithms and Simulated Annealing for Minimizing Transfer Waiting Time in Transit Systems
}

\author{
Vahid Poorjafari, Wen Long Yue, and Nicholas Holyoak
}

\begin{abstract}
Reducing the waiting time imposed on thepassengerstransferring between transit lines has always been a concern for public transport schedulers, as it is a complicated problem by nature. Typically, network-wide minimization of transfer waiting time is a highly complex optimization problem, particularly in the case of dealing with huge transit networks. This problem is unlikely to be solved by exact optimization techniques. This study aims to investigate the capability of two powerful metaheuristic algorithms, genetic algorithms and simulate annealing, in coping with the transfer optimization problem. Amathematical model is presented in this study for minimizing the total transfer waiting time in transit systems. Based on this model, a genetic algorithm and a simulated annealing algorithm are developed and applied to a transit network comprising numerous transfer points. The comparative analysis of the results revealed the ability of the both algorithms in reducing the transfer waiting time although the genetic algorithm could return better results in relatively shorter computation times.
\end{abstract}

Index Terms - Transit, public transport, simulated annealing, genetic algorithms.

\section{INTRODUCTION}

Urban transit systems usually do not provide direct services between all origins and destinations due to economic reasons. Hence, public transport users are often required to transfer between transit lines in order to complete their trips. In such conditions, poor temporal coordination between arrival and departure times of related services could lead to imposing long and unendurable waiting times on transferringpassengers, particularly when headways are relatively long. In other words, transferring between transit lines could result in long delays unless the related lines are temporally well-coordinated. This coordination is widely recognized as transit timetable synchronization in the domain of transit systems planning and scheduling.

Timetable synchronization is a crucial step in public transport timetabling,which is concerned with setting the timetables for the purpose of minimizing the waiting time for transferring passengers. Although there are several ways for timetable coordination, modification of the departure times from the first stop/depot (i.e. the start time) is the most

Manuscript received October 30, 2014; revised February 2, 2015. This work was supported in part by University of South Australia in Australia.

Vahid Poorjafari and Wen Long Yue are with the School of Natural and Built Environments, University of South Australia, Australia (e-mail: cxqvy002@mymail.unisa.edu.au,wen.yue@unisa.edu.au).

Nicholas Holyoak is with the School of Computer Science, Engineering and Mathematics, Flinders University, Australia (e-mail: nicholas.holyoak@flinders.edu.au). common method for the schedule synchronization. This process can be performed through either altering the service frequencies or keeping the pre-determined frequencies of transit lines [1]. In practice, this task is sometimes simplified in the favor of providing coordination among a limited number of lines at a few transfer points. Nonetheless, network-wide synchronization in which all feasible transfers are taken into account is unanimously considered as the most difficult task in public transport scheduling [2].

The mathematical formulation of network-wide timetable synchronization usually leads to a complex combinatorial optimization problem [1]-[3]. From a mathematical point of view, the problem complexity is mainly because of the need to search for the optimum solution in an extremely huge search space made up by permutation of all possible departure and arrival times of transit vehicles (e.g. train, bus, etc.). This problem is believed to be a combinatorial non-deterministic polynomial-time hard (NP-Hard) optimization problem, which is very unlikely to be solved with exact optimization techniques [4]. Therefore, approximate optimization techniques (e.g. heuristic and metaheuristic algorithms) that return near-optimum solutions in relatively shorter computation times could be potential alternatives for dealing with this problem.

This paper presents the application of genetic algorithms and simulated annealing, as two powerful metaheuristics, in timetable synchronization for the purpose of comparing their strength and weakness in this regard. First, the timetable synchronization is formulatedin the following sections and then, the capability of these algorithms is investigated through a numerical example.

\section{REVIEW OF THE LITERATURE}

Minimization of transfer waiting time in transit networks has always been a big concern for public transport schedulers and several methods have been proposed in the literature for improving the temporal integration of transit services. These methods are mainly different corresponding to their objectives (e.g. maximizing simultaneous arrivals, minimizing long waiting times, etc.). However, minimization of the total transfer waiting time has been the most common objective used in the previous studies. The total transfer waiting time is considered as the summation of the waiting times incurred by all transferring passengers at all transfer points in a transit network over a scheduling period (e.g. peak hour). From a mathematical point of view, most of the models proposed in the literature for transfer optimization fall within the Mixed-Integer Programming (MIP) and the 
Mixed-Integer Nonlinear Programming (MINP) classes of optimization problems. Correspondingly, different solution methods have been employed in the literature for solving the proposed models.

A MIP model was proposed in [2] for the purpose of maximizing the number of simultaneous arrivals of buses at selected transit centers using a heuristic algorithm. This model was modified in [3] in order to prevent bus bunching in urban bus systems using 'Branch and Bound' and multi-start iterated local search methods. Another approach was developed in [5] for minimizing transferwaiting time between railway lines throughan optimization-based heuristic algorithm. Reference [6] presented a model for synchronizing train lines and bus feeders using genetic algorithms. Similarly, a schedule synchronization model was proposed in [4] for minimizing the total transfer waiting time for bus networks with genetic algorithms. Reference [7] also developed a MIP model for transfer optimization in public transport systems using genetic algorithms.

In complex optimization problems, the quality of optimum solutions is highly affected by the efficiency of the selected solution method. As mentioned above, heuristic and metaheuristic (i.e. general-purpose heuristic) algorithms have been widely used in the previous studies as the solution method for the timetable synchronization problem. To the best of our knowledge, genetic algorithms have been the most-used metaheuristic for coping with this problem due to their capabilities and simplicity in application. However, application of other computational methods, such as simulated annealing, has been suggested in the previous studies, such as in [4] and [8], for the purpose of comparing the other optimization methods with genetic algorithms.This study aims to address this crucial point through comparing the effectiveness of genetic algorithms and simulated annealing in dealing with the transit timetable synchronization problem.

\section{PROBLEM Formulation}

This section presents the mathematical formulation of the transit timetable synchronization problem. Let us assume two public transport lines $i$ and $j$ intersecting at a transfer point $c$ (e.g. a transit interchange). The transfer time from $i$ to jat ccomprises the walking time $\left(w_{i j}\right)$ between the related stops or platforms (including the boarding time) and the transfer waiting time $\left(t f_{i j}\right)$ for catching the next service. While $w_{i j}$ is influenced by the physical distance between the related platforms and ease of access, $t f_{i j}$ is the direct consequence of temporal coordination between the vehicles operatingon $i$ and $j$.

The feasible transfer waiting time for transferring from $i$ to $j$ at $c$ is the interval between the arrival time of a transit vehicle in $i$ at $c\left(A_{i}\right)$ and the departure time of the vehicle in $j\left(D_{j}\right)$ departing $c$ after the time $A_{i}+w_{i j}$. Therefore, $t f_{i j}$ can be expressed as:

$$
t f_{i j}=D_{j}-A_{i}-w_{i j}
$$

The departure time of each transit vehicle from $c$ equals its arrival time at $c$ plus the dwell time $(d)$ that the vehicles spend onembarking and disembarking passengers at $c$. Thus,
Equation (1) can be written as a function of arrival times as below:

$$
t f_{i j}=A_{j}+d_{j}-A_{i}-w_{i j}
$$

As mentioned previously, there are several approaches for timetable synchronization. The most common way, which is widely used in public transport timetabling, is to set the start time (i.e. the departure time from the first stop/depot) of intersecting lines so that the waiting time for the passengers transferring between them becomes minimized. Accordingly, the model presented in this study is made based on this approach. Furthermore, the line frequencies are assumed to be kept unchanged in this model in order to avoid the need for adding or removing current services.

Considering the arrival time of transit vehicles at $c$ as the summation of their start times $(S)$ and the running time from the first stop/depot to $c(r)$, Equation (2) can be rewritten as:

$$
t f_{i j}=S_{j}+r_{j}+d_{j}-\left(S_{i}+r_{i}\right)-w_{i j}
$$

where, $S_{i}$ and $S_{j}$ are the start times of the first operating vehicles in $i$ and $j$, respectively, over the intended scheduling period.

As described in the previous section, minimization of the total transfer waiting time in a transit network is the main objective of the schedule synchronization process. Therefore, all transfer waiting times at all transfer points should be taken into consideration. Equation (3) shows that the total transfer waiting time in a network can be expressed as a function of start times. Therefore, the desired timetable attained from the synchronization process can be achieved through identifying the set of start times by which the total transfer waiting time over a scheduling period is minimized.

In order to develop the mathematical model for the timetable synchronization problem, it is assumed that all the physical characteristics of a transit network, including the lines alignment, average running times on network segments and transfer point locations are known beforehand. Moreover, it is assumed that the walking time, the dwell time and the transfer counts at each transfer point are given. The headways are also considered fixed over the intended scheduling period. Considering the total transfer waiting time as the summation of the waiting time incurred by all transferring passengers at all transfer points, the timetable synchronization model can be expressed as:

$$
\min Z=\sum_{i=1}^{N} \sum_{j=1}^{N} \sum_{c=1}^{M} \sum_{u=1}^{U} \sum_{v=1}^{V} t f_{i j}^{c u v} . P_{i j}^{c} \cdot f_{i j}^{c u v}
$$

Subject to:

$$
\begin{aligned}
& t f_{i j}^{c u v}=X_{j}^{v}+S_{j}^{v}+r_{j}^{c}+d_{j}^{c v}-\left(X_{i}^{u}+S_{i}^{u}+r_{i}^{c}\right)-w_{i j}^{c} \\
& f_{i j}^{c u v}=\left\{\begin{array}{l}
1, \text { feasible transfers } \\
0, \text { unfeasible transfers }
\end{array}\right. \\
& \quad t f_{i j}^{c u v} \geq 0 \\
& \frac{-h_{i}}{2} \leq X_{i}^{u} \leq \frac{h_{i}}{2}, \quad \forall i \in\{1, \ldots, N\}
\end{aligned}
$$




$$
\begin{gathered}
X_{i}^{u} \in \mathbb{Z}, \quad \forall i \in\{1, \ldots, N\} \\
S_{i}^{u}, r_{i}^{c}, d_{i}^{c u}, w_{i j}^{c} \geq 0, \forall i, j \in\{1, \ldots, N\}, c \in\{1, \ldots, M\}
\end{gathered}
$$

where,

$N$ : Number of lines in a transit network

$M$ : Number of transfer points in a transit network

$U, V$ : Sets of operating vehicles in a pair of intersecting transit lines over a scheduling period

$t f_{i j}^{c u v}$ : Transfer waiting time for transferring from the u-th vehicle in line $i$ to the $\mathrm{v}$-th vehicle in line $j$ at transfer point $c$

$X_{i}^{u}$ : Shift in the start time of the $\mathrm{u}$-th vehicle in line $i$

$S_{i}^{u}$ : Start time of the u-th vehicle in line $i$

$r_{i}^{c}$ : Running time from the first stop/depot in line $i$ to transfer point $c$

$d_{j}^{c v}:$ Dwell time of the $\mathrm{v}$-th vehicle in line $j$ at transfer point $c$

$w_{i j}^{c}$ : Walking time from line $i$ to line $j$ at transfer point $c$

$P_{i j}^{c}$ : Number of transferring passengers in each feasible transfer from line $i$ to line $j$ at transfer point $c$

$f_{i j}^{c u v}$ : Factor that filters unfeasible transfers when there is no feasibility of transfer from the $\mathrm{u}$-th vehicle in line $i$ to the $\mathrm{v}$-th vehicle in line $j$ at transfer point $c$

$h_{i}$ : Headway of line $i$

The objective function of this model (Equation (4)) represents the total transfer waiting time in a transit network over a scheduling period. This model is a new form of the timetable synchronization model presented in [4] modified based on arrival times. In this model, $X$ is selected as the decision variable representing the required shift in the existing start times (i.e. current timetables) for minimizing the total transfer waiting time. This variable takes only integer values (Constraint 9) since transit timetables are usually set in minutes. Consequently, this mathematical programming model falls within the Integer Programming (IP) class of optimization problems. The decision variable $X$ is allowed to vary within the range $[-h / 2, h / 2]$ (Constraint 8 ) in order to keep the existing line frequencies.

\section{ApPlicAtion OF MEtAHEURistics}

The optimization model presented in the previous section is a complex optimization problem by nature. The main reason for its complexity is that there are many feasible combinations of shifts in the start times of vehicles in a transit network. In other words, the start times of transit lines can be set in numerous ways and the optimum solution has to be found through exploring a huge search space made up of all feasible solutions. This search becomes practically impossible in the case of dealing with large transit networks consisting of tens of intersecting lines and transfer points. In fact, this model is a combinatorial optimization problem whose complexity increases exponentially by the network size. Such optimization problems are unlikely to be solved to optimality within polynomially-bounded computation time and the only possibility for coping with such problems is to trade optimality for efficiency through employing approximate solution techniques [9].

Typically, there are two possible approaches for dealing with difficult combinatorial optimization problems: problem-specific heuristics and metaheuristics [10]. The major deficiency of problem-specific heuristics is that they are designed for specific problems and consequently, they are usually inapplicable to other problems. However, metaheuristics are general algorithmic frameworks that can be applied to a range of optimization problems with relatively minor modifications. Metaheuristic algorithms, which have drawn the attentions in recent years, are significantly capable and effective methods in tackling many types of complex optimization problems [11]. These methods have beensuccessfully used for solving difficult combinatorial optimization problems in operations research, as well as in different areas of engineering [12]. Hence, these algorithms could be potential alternatives for coping with the timetable synchronization problem presented in the previous section.

Metaheuristics include a range of algorithms with different characteristics. Generally, determination of the most appropriate metaheuristic for an optimization problem is a hard task [10]. In other words, the choice of an efficient metaheuristic is an experience-dependent process that requires extensive analyses on different metaheuristics and their basic parameters and operators. In this study, genetic algorithms and simulated annealing are selected for tackling the proposed timetable synchronization problem.

\section{A. Genetic Algorithms (GAs)}

Genetic algorithms are population-based metaheuristics inspired by the way species evolve and adapt to the environment. These algorithms are iterative search techniques in which each solution is called a chromosome and defined as a string of values (i.e. genes) that the problem variables can take. Each solution is formed by combining two predecessor solutions. A number of solutions are generated in each generation and assessed based on their fitness values. Then, the solutions evolve from one generation to the next generation through applying three principal operators, namely, selection, crossover and mutation. This process continues until the (near) optimum solution is found. The successful application of GAs to diverse combinatorial problems has proved the capability of these metaheuristics in coping with this class of optimization problems [12].

\section{B. Simulated Annealing (SA)}

Simulated annealing is a single-solution metaheuristic widely used for dealing with discrete, as well as continuous optimization problems. This algorithm is inspired by an analogy between combinatorial optimization problems and the physical annealing of solids [9]. SA is an iterative search method that starts from a randomly generated initial solution. A trial solution is generated in each iteration using a neighborhood creating function. If the trial solution is better than the current solution, it is chosen as the current solution. However, if the trial solution is worse than the current solution, the trial solution is accepted with a probability that depends on the difference in the objective function value and a control parameter called temperature. This probability for minimization problems is defined based on Metropolis distribution as below [9]:

$$
P_{\text {accept }}\left(s, s^{\prime}, T\right)= \begin{cases}1, & \text { if } f\left(s^{\prime}\right)<f(s) \\ \exp \left(\frac{f(s)-f\left(s^{\prime}\right)}{T}\right), & \text { otherwise }\end{cases}
$$


where, $P_{\text {accept }}$ is the probability of acceptance, $s$ is the current solution, $s$ ' is the trial solution, $T$ is the temperature, and $f(s)$ and $f\left(s^{\prime}\right)$ are the objective function values under the current and the trial solutions, respectively. After sufficient iterations at each temperature, the temperature is lowered based on anannealing schedule (i.e. cooling scheme) in order to decrease the probability of accepting worse trial solutions.

The key feature of simulated annealing algorithm is its ability in escaping local optima and searching for the global optimum in the search space [11]. It has been observed that $\mathrm{SA}$ is capable to return excellent results for a range of complex and big-size optimization problems [10].

\section{Comparative ANALYSIS OF GA AND SA}

In order to compare GA and SA in terms of dealing with the timetable synchronization problem in this study, their capabilities are examined through a numerical example. The example is a hypothetical transit networkconsisting of 20 bidirectional lines, as shown in Fig 1. In this network, 10 East-West lines (lines 1-10) intersect 10 North-South lines (lines 11-20) at 100 transfer points (C1-11 to C10-20). In other words, 800 possible transfer approaches (i.e. 8 approaches at each transfer point) are considered in this example, which sufficiently increase the complexity of the synchronization problem. The headways are considered in the range of 6 to 15 minutes and they are assumed to be fixed over the scheduling period.

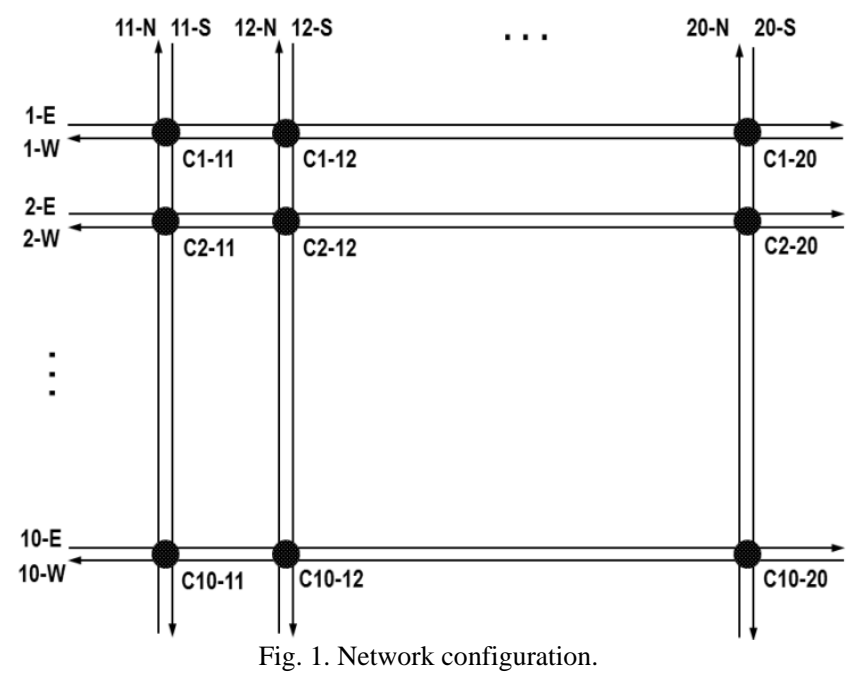

For the sake of complexity, the network is assumed dissymmetric so that the average running times on network segments (i.e. travel time between transfer points) are assumed different, in the range of 1 to 2.5 minutes. The number of transferring passengers between each pair of the intersecting lines is also considered between 0 and 10 passengers. In this example, the walking time and the dwell time at all the transfer points are assumed as 30 seconds.

In this example, the impact of timetable synchronization on transfer waiting time is investigated through two scenarios, namely, uncoordinated and synchronizedtimetables. Under the uncoordinated timetable, all the transit vehicles are assumed to depart the first stop/depot at the same time (8:00 am) and it is intended to create the synchronized timetable through modifying the line start times using the proposed optimization model. Also for the purpose of comparing the metaheuristics, the same formis consideredfor solutions inGA and SA. Each solution is defined as a vector of 40 integer values representing the shifts in the lines start time $\left(X_{i}\right)$. A genetic algorithm and a simulated annealing algorithm were developed using Matlab programming language based on the model presented in Section III.

Since the effectiveness of metaheuristics is sensitive totheir basic parameters and operators, a range of sensitivity analyses were performedto determine the most appropriate settings for these algorithms. In terms of the genetic algorithm, the analysis was conducted on population size (i.e. the number of trial solutions in each generation) and crossover fraction (i.e. the proportion of children in the population). Three population sizes (20, 40 and 60), as well as five crossover fractions $(0.5,0.6,0.7,0.8$ and 0.9$)$ were considered for the genetic algorithm and the quality of the best solution was examined under 15 settings made up of the combinations of these values.

Fig. 2 displays the variation of the optimum solution found by the genetic algorithm under thesesettings. As shown in this illustration, the total transfer waiting time in the network varied under the different GA settings. However, the minimum transfer waiting time in the network was found by the GA when the population size is 40 and the crossover fraction is 0.9 . Under this GA setting and using a $1.83 \mathrm{GHz}$ CPU, the timetable synchronization model was solved for the intended networkin 847 seconds, after 99 generations. Fig. 3 demonstrates the improvement in the objective function value for this solution.

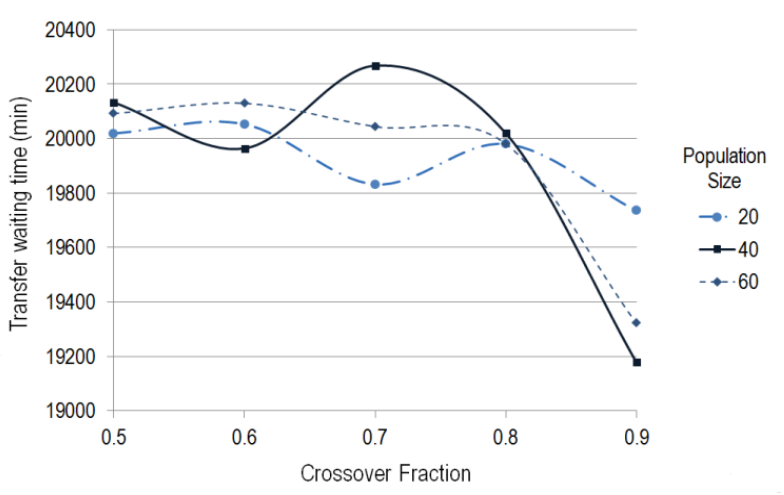

Fig. 2. Sensitivity analysis on the GA parameters.

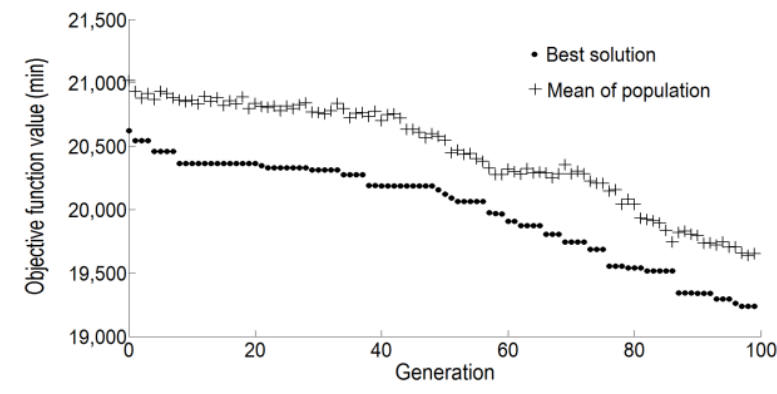

Fig. 3. Improvement in the objective function value by generations for the best solution found by GA.

Similarly, a sensitivity analysis was executed on the simulated annealing algorithm in order to adjust its basic parameters. Since the annealing schedule has significant effects on SA performance, in this analysis the main attention 
was paid on the initial temperature, as well as on the cooling scheme (i.e. the annealing schedule). After some preliminary evaluations, three different values $(50000,100000$ and 150000) were assumed for the initial temperature. Three different cooling schemes, comprising Fast Cooling Scheme (FCS), Exponential Cooling Scheme (ECS) and Logarithmic Cooling Scheme (LogCS), were also considered for the simulated annealing algorithm.The temperature decrementing functions used in these schemes are considered as follows:

Fast cooling scheme:

$$
T_{n}=\frac{T_{0}}{n}
$$

Exponential cooling scheme:

$$
T_{n}=T_{0} \cdot\left(0.95^{n}\right)
$$

Logarithmic cooling scheme:

$$
T_{n}=\frac{T_{0}}{\ln (n)}
$$

where, $T_{n}$ is the temperature at $n$-th stage and $T_{0}$ is the initial temperature. The results of the sensitivity analysis on the different SA settings are shown in Fig. 4. As shown in this figure, the best solution found by SA was under the exponential cooling scheme when $T_{0}=100000$. Using this setting, the problem was solved after 6002 iterations in 1439 seconds. Fig. 5 shows the improvement in the objective function value by iterations for the SA algorithm.

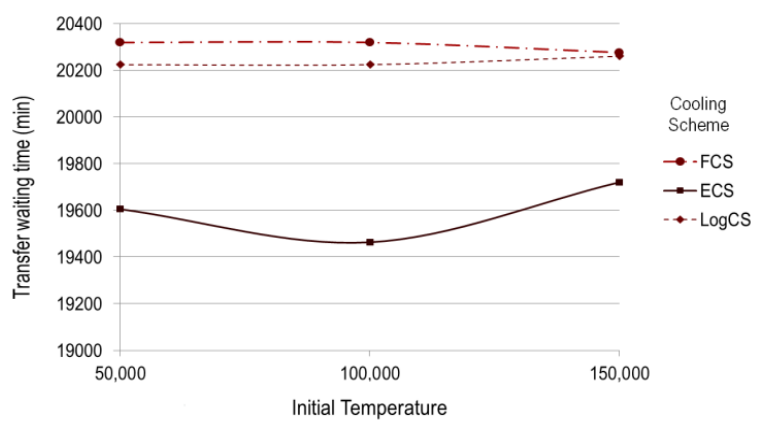

Fig. 4. Sensitivity analysis on the SA parameters.

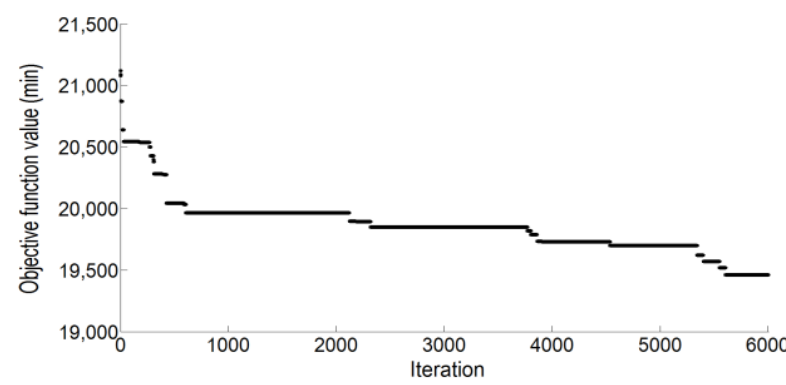

Fig. 5. Improvement in the objective function value by iterations for the best solution found by SA.

The comparison between the best resultsattained by GA and SA revealed that the genetic algorithm returned a better optimum solution in a shorter computation time. The objective function value (i.e. the total transfer waiting time in the network) for the best solution found by the simulated annealing was 19463 minutes. However, the genetic algorithm yielded a better optimum with the objective function value of 19178 minutes. GA found this optimum in almost $60 \%$ of the computation time that SA spent on finding the best solution. In other words, the genetic algorithm was able to return a better result in a shorter computation time, compared to the simulated annealing algorithm.

Using the best solution found by GA, the start times of the transit lines were modified and the synchronized timetable was created. Table I presents the comparison of the uncoordinated and the synchronized timetables for one hour of the scheduling period (8:00-9:00 am). The total transfer waiting time in the network fell by $9.2 \%$, from $21121 \mathrm{~min}$ to $19178 \mathrm{~min}$, under the synchronized timetable. In fact, temporal coordination of the transit services in the intended network resulted in a 1943 minute decrease in the waiting time, in just over one hour of the scheduling period. This equals to an average reduction of $0.5 \mathrm{~min}$ (from $5.45 \mathrm{~min}$ to $4.95 \mathrm{~min}$ ) in each passenger's travel time.It should be noted that the decrease in the travel time was not the same at all the transfer points, as expected. However, the process of timetable synchronization led to a noticeable reduction in the waiting times incurred by the transferring passengers in this transit network.

TABLE 1: IMPACTS OF TIMETABLE SYNCHRONIZATION

\begin{tabular}{c|c|c|c|c}
\hline \hline \multicolumn{2}{c|}{ Network Section } & $\begin{array}{c}\text { E-W } \\
\text { Lines }\end{array}$ & $\begin{array}{c}\text { N-S } \\
\text { Lines }\end{array}$ & $\begin{array}{c}\text { Entire } \\
\text { Network }\end{array}$ \\
\hline \hline \multirow{2}{*}{ Transfer counts } & 1999 & 1876 & 3875 \\
\hline \multirow{2}{*}{$\begin{array}{c}\text { Uncoordinated } \\
\text { timetable }\end{array}$} & $\begin{array}{c}\text { Total } t f \\
\text { (min) }\end{array}$ & 11168 & 9953 & 21121 \\
\cline { 2 - 5 } & $\begin{array}{c}\text { Ave. } t f \text { for each } \\
\text { passenger } \\
(\text { min) }\end{array}$ & 5.59 & 5.31 & 5.45 \\
\hline \hline \multirow{2}{*}{$\begin{array}{c}\text { Synchronized } \\
\text { timetable }\end{array}$} & $\begin{array}{c}\text { Total } t f \\
\text { (min) }\end{array}$ & 9926 & 9252 & 19178 \\
\cline { 2 - 5 } & $\begin{array}{c}\text { Ave. } t f \text { for each } \\
\text { (min) }\end{array}$ & 4.97 & 4.93 & 4.95 \\
\hline \hline
\end{tabular}

\section{CONCLUSION}

In this paper, we presented a comparison between genetic algorithms and simulated annealing, as two widely-used and powerful metaheuristics, in terms of coping with the transit timetable synchronization problem. A mathematical model was presented for the purpose of minimizing the total waiting time in public transport systems. The model aims to minimize the waiting time imposed on the passengers transferring between transit lines through modifying the lines start time.

A genetic algorithm and a simulated annealing algorithm were developed in this study in order to solve the proposed optimization problem. A hypothetical transit network consisting of numerous transfer points was also considered as a complicated case for the purpose of comparing the 
capability of these algorithms. The algorithms were fitted to the proposed optimization model through conducting a range of sensitivity analyses on their principal parameters and operators. Afterwards, their performance on solving the problem was examined through a comparative analysis. The outcome showed that both of the algorithms are able to tackle the problem and reduce the total transfer waiting time. However, the genetic algorithm could return better results in a shorter computation time.

The domain of metaheuristics includes a range of algorithms which are capable to deal with different types of hard optimization problems.Therefore, future work could involve the application of other metaheuristics to the transit schedule synchronization problem, which is recognized as a complex optimization problem. Further investigations on the other GA and SA parameters and operators could also be an extension for this study.

\section{REFERENCES}

[1] V. Guihaire and J. K. Hao, "Transit network design and scheduling: A global review," Transportation Research Part A: Policy and Practice, vol. 42, no. 10, pp. 1251-1273, 2008.

[2] A. Ceder, B. Golany, and O. Tal, "Creating bus timetables with maximal synchronization," Transportation Research Part A: Policy and Practice, vol. 35, no. 10, pp. 913-928, 2001.

[3] O. J. I. Rojas and Y. A. R. Solis, "Synchronization of bus timetabling,"Transportation Research Part B: Methodological, vol. 46 , no. 5, pp. 599-614, 2012.

[4] F. Cevallos and F. Zhao, "Minimizing Transfer Times in a Public Transit Network with Genetic Algorithm,"Transportation Research Record: Journal of the Transportation Research Board, vol. 1971, no. 1, pp. 74-79, 2006

[5] R. C. W. Wong and J. M. Y. Leung, "Timetable synchronization for mass transit railway," presented at the 9th International Conference on Computer-Aided Scheduling of Public Transport (CASPT), San Diego, 2004.

[6] P. Shrivastava and S. L. Dhingra, "Development of coordinated schedules using genetic algorithms," Journal of Transportation Engineering, vol. 128, no. 1, pp. 89-96, 2002.

[7] Y. Shafahi and A. Khani, "A practical model for transfer optimization in a transit network: Model formulations and solutions," Transportation Research Part A: Policy and Practice, vol. 44, no. 6, pp. 377-389, 2010.

[8] V. Poorjafari, W. L. Yue, and N. Holyoak, "Application of simulated annealing in transit schedule synchronization," International Journal of Modelling and Optimization, vol. 4, no. 6, pp. 476-481, 2014.

[9] M. Dorigo and T. Stutzle, Ant Colony Optimization, US: The Massachusetts Institute of Technology, 2004, pp. 23-49.
[10] J. Dreo, A. Petrowski, P. Siarry, and E. Taillard, Metaheursitics for Hard Optimization, Germany: Springer-Verlag Berlin Heidelberg, 2006, pp. 16-19.

[11] F. Glover and G. A. Kochenberger, Handbook of Metaheuristics, US Kluwer Academic Publishers, 2003, ch. 10, pp. 287-291.

[12] M. Gendreau and J. Y. Potvin, "Metaheuristics in combinatorial optimization," Annals of Operations Research, vol. 140, no. 1, pp $189-213$.

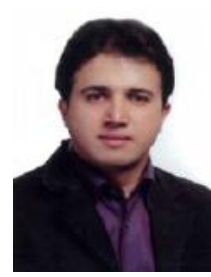

Vahid Poorjafari is a $\mathrm{PhD}$ candidate of transport engineering at the School of Natural and Built Environments, University of South Australia. His research interests include transit planning, urban transportation, transport infrastructure design, system analysis and optimization in transportation systems. He has several publications on different aspects of transportation planning and engineering.

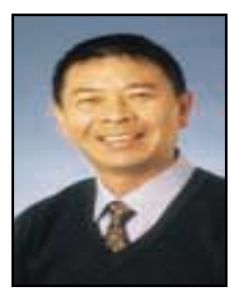

Wen Long Yue is a senior lecturer and the program director of transport engineering at the School of Natural and Built Environment, University of South Australia. His professional activities, industry consultancy activities, teaching, academic research and research student supervision interests span in all the aspects of transport systems engineering. He is an expert in simulation modeling for transport systems and parking systems. He is a lecturer in transpor planning and traffic engineering at postgraduate and undergraduate levels. His research interests include the safety and operational characteristics of signalized intersections, the analysis procedures of accidents, the operation of public transport systems, transport survey design, logistics operation and management.

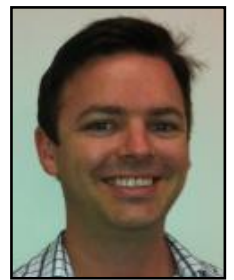

Nicholas Holyoak is a research fellow with Flinders University specializing in the transport systems field. His research interests include travel behavior, modeling travel demand, network analysis, survey design, deployment and data analysis, sustainable transport and electric vehicles. He currently lectures in the fields of road design and traffic management. 